\title{
Lo que necesita este país es más internacionalistas: el recurso a la serie animada Los Simpson para la enseñanza del derecho internacional
}

\author{
Luciano Pezzano: $\quad$ Universidad Nacional de Córdoba, UNC \\ Leopoldo M. A. Godio: $\quad$ Universidad de Buenos Aires, UBA
}

Recibido: 08/01/2021

Aceptado: $18 / 05 / 2021$

Publicado: 08/09/2021

Resumen: El artículo propone analizar la utilización de la conocida serie animada de televisión Los Simpson como instrumento multifuncional para el diseño de nuevas estrategias metodológicas dedicadas a la enseñanza del derecho internacional. Sobre la base de que su empleo con fines pedagógicos en otras disciplinas ha sido bien documentada $-y$ dado el impacto en la cultura popular de nuestro país que ha marcado a varias generaciones de estudiantes-, la propuesta plantea que la riqueza de la trama de Los Simpson abunda en ejemplos que permiten acercar a los estudiantes a las más diversas cuestiones abordadas en el derecho internacional público, así como desarrollar actividades íntegramente centradas en torno a episodios de la serie, de las que se aporta un caso concreto.

Palabras clave: Metodología de la enseñanza, derecho internacional público, cultura popular, Los Simpson.

* Cómo citar este artículo: Godio, L. M. A. y Pezzano, L. (2021). Lo que necesita este país es más internacionalistas: el recurso a la serie animada "Los Simpson" para la enseñanza del derecho internacional. Relaciones Internacionales, 30(61), 119.

https://doi.org/10.24215/23142766e119

Editor: Juan Alberto Rial, Instituto de Relaciones Internacionales Facultad de Ciencias Jurídicas y Sociales (Universidad Nacional de La Plata)
Entidad editora: Relaciones

Internacionales, es una publicación del Instituto de Relaciones Internacionales (Facultad de Ciencias Jurídicas y Sociales (Universidad Nacional de La Plata Argentina)

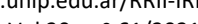




\title{
This country needs more internationalists: using the ani- mated series "The Simpsons" to teach international law
}

\section{Luciano Pezzano", Leopoldo M. A. Godio**}

\begin{abstract}
This paper aims at discussing the use of the well-known animated television series The Simpsons as a multifunctional instrument for the design of new methodological strategies to teach international law. Given that its use for pedagogical purposes in other disciplines has been well documented -and since its impact on our country's popular culture has marked several generations of students-, this proposal suggests that the richness in The Simpsons' plot provides lots of examples that bring students closer to the most diverse issues addressed in public international law. The series also allows for the development of activities entirely focused on some of its episodes, from which a specific case is provided.
\end{abstract}

Key-words: Teaching methodologies, Public International Law, popular culture, The Simpsons.

\footnotetext{
* Abogado (UCES, San Francisco), Magister en Relaciones Internacionales (CEA-UNC) y Doctor en Derecho y Ciencias Sociales (UNC). Profesor Ayudante " $A$ " de Derecho Internacional Público (UNC). Profesor Asociado de Derecho Internacional Público y de la Integración (UCES, San Francisco). Miembro Asociado de AADI. Miembro del Instituto de Derecho Internacional Público y de la Integración de la Academia Nacional de Derecho y Ciencias Sociales de Córdoba.

** Abogado, Magíster en Relaciones Internacionales y Doctor en Derecho (UBA). Investigador adscripto del Instituto de Investigaciones Jurídicas y Sociales “Ambrosio L. Gioja”. Profesor de Derecho Internacional Público (UBA-UCA-UP-USI). Miembro Titular de AADI; Miembro Consultor del CARI y Miembro del Instituto de Derecho Internacional de la Academia Nacional de Derecho y Ciencias Sociales de Buenos Aires.

Nota de los autores: para referirnos a los episodios de la serie, utilizaremos el título fiel a la denominación con la cual se le conoce en América Latina, con la aclaración de su original en inglés y entre paréntesis. Ante cualquier duda $-\mathrm{y}$ recuerden que sólo podéis hacer tres preguntas- nuestro contacto es luciano.pezzano@unc.edu.ar / leopoldogodio@derecho.uba.ar, esperamos haberles iluminado.
} 


\section{Introducción}

Uno de los mayores desafíos actuales de la docencia -al cual no escapa el nivel universitario de grado- consiste en atraer la atención de los estudiantes y su dificultad se incrementa particularmente en las ciencias jurídicas, un ámbito en el cual las estrategias pedagógicas de las universidades argentinas poseen una impronta de tradición formalista, con escasa atención en otras ciencias sociales y baja profesionalización del rol docente, debido a la preocupación marginal que presentaban estos aspectos -posiblemente esta afirmación se deba a que la enseñanza tradicional ha formado al abogado para un estilo de litigio en el que las normas constituyen la justa respuesta a todas las preguntas (Böhmer, 2003: 18)- al punto de provocar que los docentes reproduzcan, en general, el mismo estilo pedagógico recibido durante su etapa de alumnos universitarios (Bergoglio, 2006: 109), caracterizado principalmente por la "clase magistral" y la orientación de la enseñanza basada en libros y no en el derecho como experiencia de interacción social (Fucito, 2000: 94 y 336).

Esta configuración metodológica-pedagógica, fuertemente arraigada en la enseñanza del derecho -no aplicable exclusivamente a las universidades argentinas, sino también a establecimientos latinoamericanos donde el desarrollo de cursos enteros se organiza, predominantemente, alrededor de clases monologadas, en las que el profesor deriva y elabora comentarios aclaratorios, argumentativos e interpretativos con una gran formación teóricadificulta la introducción de estrategias innovadoras y alternativas orientadas a la participación activa y “a partir" del estudiante (Lista, 2006: 256-257).

Lo anterior ha permitido que especialistas en educación concluyan en la prevalencia "de un estilo de pensamiento jurídico fuertemente formalista (...) [que] ha impregnado, durante mucho tiempo, planes y programas de estudio, textos y estilos de trabajo jurídico" (Bergoglio, 2006: 122).

Ante este cuadro de situación es que los docentes deben recurrir a estrategias pedagógicas tendientes a establecer puentes y vínculos con los estudiantes de leyes, a fin de incrementar la efectividad de sus clases y lograr, como resultado, una adecuada elaboración y transmisión de conocimiento que supere el clásico proceso de enseñanza-aprendizaje. Aquí es donde la utilización de la cultura popular habilita al debate y razonamiento conjunto orientado hacia una "construcción compartida de conocimiento", generado a partir de supuestos iniciales de carácter no-jurídicos y en un ámbito o espacio en el cual el estudiante se sienta protagonista y confiado para intervenir en una dinámica que, de presentarse de otro modo, le resultaría más dificultosa.

En ese sentido, pensamos que la utilización de alternativas metodológicas que aprovechen la cultura popular -en particular, películas y series de televisión (re)conocidas por los estudiantes- para vincularla a los contenidos propios de una asignatura, como el derecho internacional público, puede resultar beneficioso para la enseñanza y fijación del conocimiento por parte de los estudiantes.

En efecto, se ha advertido la existencia de una íntima relación entre las leyes, los abogados y la cultura popular, donde esta última transmite a un público -que generalmente ignora los asuntos jurídicos- información distorsionada respecto de las normas aplicables a un supuesto imaginario, excepto en los contados casos de individuos que siguen la actividad 
judicial, administrativa y legislativa (Ho, 2003: 275) que, en su adaptación local en Argentina, podría contar con un equivalente en el Boletín Oficial. En idéntico sentido, Keslowtiz afirma que la cultura popular impacta, habitualmente, en el desarrollo de áreas específicas de las normas jurídicas debido a la influencia que ejercen en la población, a pesar de contar con representaciones inexactas y la tendencia de los legisladores -como también, de eventuales operadores judiciales en sistemas comparados- para complacer la voluntad de sus electores (Keslowtiz, 2008: 2788).

No creemos -como tal vez podría prejuzgarse- que el diseño de esta estrategia constituya una banalización o un menoscabo en la jerarquía y contenidos de una materia tan importante como es el derecho internacional público. Por el contrario, se trata de una forma de lograr transmitir conocimiento a través de las propias emociones y vivencias, para de este modo acercar a los estudiantes a los textos jurídicos.

Es así que nos proponemos analizar la utilización de una producción animada de televisión en particular, una verdadera herramienta de la cultura popular y cuya notoria difusión resulta de difícil omisión: la conocida serie animada de televisión Los Simpson, un verdadero instrumento multifuncional para el diseño de nuevas estrategias metodológicas dedicadas a la enseñanza del derecho internacional ${ }^{1}$.

Para ello cabe preguntarnos ¿por qué Los Simpson? Su utilización con fines pedagógico-sociales ha sido documentada y su éxito, analizado en establecimientos de educación media de Alemania (Terrell, 2014/2015: 6-7) y EE.UU., dos culturas claramente diferentes entre sí, al punto que se llegó a considerar la serie animada como un complemento filosófico de enseñanza que enfatiza el uso de la imaginación aplicado a la observación de la vida cotidiana, fomentando una mayor comprensión de los estudiantes en general e incentivando la discusión crítica y efectiva, para luego utilizar ese conocimiento más allá del aula (Scanlan \& Feinberg, 2000: 127).

La siguiente pregunta es ¿̇es posible utilizar a Los Simpson para enseñar exitosamente derecho internacional público? En las siguientes líneas expondremos los distintos argumentos que permiten dar respuesta a ambos interrogantes para, posteriormente, concluir respecto de su validez didáctica y aportar algunas reflexiones adicionales.

\footnotetext{
${ }^{1}$ En el presente trabajo nos limitaremos a analizar aquellos episodios que tratan aspectos propios del derecho internacional público y excluiremos los referidos al derecho internacional privado, sin que ello signifique carencias de ejemplos para la última especialidad -como lo es el episodio dedicado a una adopción internacional, protagonizada por Selma Bouvier, quien decide adoptar a una menor nacida en China y requiere, para lograrlo, la colaboración de su cuñado Homero. Cfr. "Aventura en China” (Goo Goo Gai Pan), temporada 16, episodio 18.
} 


\section{La serie animada Los Simpson como herramienta didáctica y su carác- ter multifuncional}

Los episodios de Los Simpson -aunque podríamos incluir referencias a la serie Futurama y la más reciente creación de Matt Groening (Des)Encanto ${ }^{2}$ - cuentan con una gran cantidad de sátiras situaciones basadas en hechos históricos o hipotéticos con una cuota de posibilidad, aunque ello no significa que sean correctos desde el rigor académico, jurídico, matemático, científico, médico, propiamente histórico o cualquiera sea la disciplina que pueda dar respuestas a sus imprecisiones ${ }^{3}$. Sin embargo, su éxito radica en la comedia diseñada con el objeto de mostrar un espejo de nuestra sociedad e incluso de nosotros mismos.

Por esta razón se explica parte de su referencia permanente por más de una generación. La serie ha llegado, incluso, a ser utilizada como parte de las consideraciones para resolver un caso judicial en los EE.UU., que mereció posteriores y elogiosos comentarios en prestigiosas publicaciones académicas (Keslowitz, 2008: 2821-2822). Es por ello que nuestro convencimiento para elegir a la familia amarilla de Springfield como metodología de enseñanza se encuentra justificado independientemente de los gustos personales, al margen del fanatismo de quienes escribimos estas líneas. En suma, lo anterior no resulta casual y existen, al menos, tres motivos que justifican nuestra utilización didáctica para transmitir conceptos del derecho internacional.

El primero es que, en sus más de tres décadas de emisión, a través de treinta y dos temporadas y más de setecientos episodios ${ }^{4}$, han cubierto una innumerable variedad de tópicos ${ }^{5}$, muchos de ellos de directo interés para el derecho internacional y que resultan de utilidad para referirse, a título ejemplificativo, a temas centrales de nuestra asignatura, como: el fundamento de los privilegios e inmunidades diplomáticos, el estatus jurídico de

\footnotetext{
${ }^{2}$ Estrenada en la plataforma Netflix, ya en su primera temporada presenta referencias expresas al derecho internacional. Por caso, en su segundo episodio los personajes principales participan de una fiesta de "despedida de soltero" y alquilan un buque para dirigirse a "mar abierto, donde todo está permitido". Asimismo, en su séptimo episodio se advierte un desarrollo y aplicación de las reglas de cortesía ceremonial en un contexto de relaciones diplomáticas oficiales entre dos reinos. Sin dudas, tanto (Des)Encanto como Futurama constituyen una invitación para realizar un trabajo similar a futuro.

${ }^{3}$ Luccasen y Thomas realizan un análisis sobre la utilización de la misma serie para enseñar economía a partir de, por ejemplo, cuando Homero Simpson descubre que posee un hermano mayor, Herb Powell, un empresario automotriz al que visita luego en Detroit (Luccasen \& Thomas, 2010: 136-149).

${ }^{4}$ Suman hasta la fecha -6 de enero de 2021- 695 episodios, a los que deben adicionarse los 48 cortos originales, una película cinematográfica y 8 videojuegos en distintas plataformas.

${ }^{5}$ Prueba de ello es la abundante bibliografía que vincula a la serie con diferentes disciplinas que comprenden la filosofía, las ciencias duras y la numismática, entre otras. Al respecto ver, respectivamente: IRWIN, W., CONARD, M. T. \& SKOBLLE, A. J. (2009), Los Simpson y la Filosofía, Barcelona, Blackie Books; SÁNCHEZ, C. H. (2010), Todo lo que sé de ciencia lo aprendí mirando Los Simpson, Buenos Aires, Ed. del autor; y, COZzETTI, M. (2011), “Bart, el coleccionista", El Reverso, № 8, pp. 4-5.
} 
los locales de la misión diplomática y el concepto superado de la extraterritorialidad ${ }^{6}$; la Organización de las Naciones Unidas ${ }^{7}$; la Corte Internacional de Justicia ${ }^{8}$; las excepciones a la aplicación espacial en materia de tratados ${ }^{9}$; el derecho del mar respecto de la jurisdicción en la zona económica exclusiva (en adelante, "ZEE") y en la alta mar $^{10}$; la clasificación de los buques públicos ${ }^{11}$; el régimen jurídico aplicable a las estructuras e islas artificiales en el mar $^{12}$; y la remoción de restos náufragos y la protección del medio marino ${ }^{13}$, para mencionar solo algunos ${ }^{14}$.

A la enumeración anterior es posible agregar que dos de ellos poseen gran interés para los profesores y estudiantes argentinos, ya que refieren a aspectos que tratan la denominada Cuestión Malvinas. Desafortunadamente, ambos episodios merecen comentarios, los que trataremos según el orden cronológico de su aparición.

La primera referencia aparece durante pocos segundos en un "especial de horror" que nos sitúa en una pesadilla de Lisa. La historia se inicia en Marruecos, donde Homero encuentra una tienda que vende una "mano de mono muy antigua" con el poder de conceder deseos a sus dueños, pero a costa de una desgracia. Como es de prever Homero, adquiere el objeto. Cuando Lisa expresa su deseo, pide por "la paz mundial” -un pedido egoísta, según Homero. La primera imagen inmediata es un anuncio con una clara imagen de referencia a la O.N.U., donde un representante del Reino Unido se disculpa con su par argentino, quien

\footnotetext{
6 “Bart contra Australia” (Bart vs. Australia), temporada 6, episodio 16.

7 “Mi querido Capitán Simpson" (Simpson Tide), temporada 9, episodio 19, entre otros.

8 "El musical de la escuela primaria" (Elementary School Musical), temporada 22, episodio 1.

${ }^{9}$ Por ejemplo, al referirse a las fórmulas de exclusión parcial del territorio de un Estado en el momento de concluir un tratado internacional, tal como lo autoriza el artículo 29 de la Convención de Viena de 1969, referida al ámbito territorial de los tratados, donde resulta útil la expresión "excepto Alaska y Rancho Viejo" (aunque en el episodio original en inglés, el Presidente de Aerolíneas Payaso manifiesta "excepto Alaska y Hawaii”). Fuente: "Miedo a volar" (Fear of Flying), temporada 6, episodio 11.

10 "La familia mansión" (The Mansion Familiy), temporada 11, episodio 12.

11 “Mi querido capitán Simpson" (Simpson Tide), temporada 9, episodio 19

12 "Exploradores a fuerza" (Boy-Scoutz $N$ the Hood), temporada 5, episodio 8.

13 "Bart en la Feria" (Bart Carny), temporada 9, episodio 12. También aparece otra referencia a la protección del medio marino durante el capítulo "El heredero de Burns", (Burns' Heir), temporada 5, episodio 18, cuando el Sr. Burns evoca momentos de su vida y entre sus recuerdo aparece un buque de Greenpeace dedicado a sabotear un vertimiento de desechos radiactivos en el mar, que fracasa al ser "saboteados desde dentro" por un joven Burns.
}

${ }^{14}$ Otros internacionalistas han analizado -indirectamente- la incidencia de la serie Los Simpsons en función del principio de no-intervención, con particular referencia a México y Argentina. Cfr. JAMNEJAD, M. \& WOOD, M. (2009) “The Principle of Non-intervention”, Leiden Journal of International Law, v. 22, p. 374, nota no 126. 
le responde "ya habrá tiempo de recuperarnos"15.

La situación pasaría desapercibida de no ser porque en el episodio en inglés advertimos que, textualmente, el primer funcionario expresa "Sorry about the Falklands" (perdón por las Malvinas) al tiempo que el argentino responde "Oh, forget it. We knew they were yours" (Oh, olvídalo. Nosotros sabíamos que eran suyas). Se trata, sin duda, de una utilización lamentable de la disputa de soberanía para intentar legitimar el reclamo del Reino Unido a través de la reiteración de la versión difundida por su Foreign Office y sintetizada por Graham Pascoe y Peter Pepper en su folleto del año 2008, "Más allá de la historia oficial. La verdadera historia de las Falklands/Malvinas". Estas estrategias, como bien señalan Kohen y Rodríguez, se tratan de argumentos y supuestos "hechos" que contradicen de manera flagrante las posiciones adoptadas por la potencia colonial a lo largo de la disputa de soberanía y constituyen un intento de reescribir la historia (Kohen \& Rodríguez, 2015: 19) ${ }^{16}$.

La segunda referencia a la Cuestión Malvinas consta de unos pocos segundos en el episodio titulado "El heredero de Burns". En una de las visitas que hace Bart a la casa del Sr. Burns, el niño expresa su deseo de cenar una pizza entregada por Krusty (el payaso) en persona. Cuando llega el pedido, Bart le pregunta a su héroe cómo es posible aquella situación cuando, al mismo tiempo, se emitía su propio programa "en vivo" y la respuesta del payaso fue, simplemente, que se trataba de un viejo programa grabado y que nadie lo advertía. Inmediatamente, la antigua transmisión proyecta un momento en el cual Krusty deja de hacer malabares para leer un comunicado y afirma “(...) niños no se alarmen, tomaron las Islas Malvinas, repito, han tomado las Islas Malvinas... Las Islas Malvinas están aquí, cerca de la costa de Argentina..."17.

Más allá de la inexactitud del comentario, ya que las Malvinas son argentinas y su expresión bienintencionada no expresaría preocupación, lo cierto es que el capítulo en inglés original utiliza -nuevamente- la expresión “invasión”, una palabra bastante menos que feliz, aunque al menos se reconoce que son islas "en disputa". Textualmente, el diálogo de Krusty afirma “(...) children, remain calm. The Falkland Islands have just been invaded. I repeat, the Falklands have just been invaded! The disputed islands lie here, off the coast of Argentina" (niños, mantengan la calma. Las Islas Falkland/Malvinas acaban de ser invadidas. Repito, ilas Malvinas acaban de ser invadidas! Las disputadas islas se encuentran aquí, frente a la costa de Argentina).

Asimismo, también podemos advertir la presencia de temas generales susceptibles de

\footnotetext{
15 “Especial de día de brujas de los Simpson II" (Treehouse of Horror II), temporada 3, episodio 7.

${ }^{16}$ También puede encontrarse una respuesta a esta estrategia del Reino Unido en BERASATEGUI, V. E. (2011), Malvinas. Diplomacia y Conflicto Armado, Buenos Aires, Proa.

17 “El heredero de Burns", (Burns' Heir), temporada 5, episodio 18
} 
una mirada jurídica y política internacional en materia de derechos humanos ${ }^{18}$, medio ambiente $^{19}$, uso de la fuerza ${ }^{20}$, terrorismo ${ }^{21}$, desarme ${ }^{22}$, las consecuencias de la Segunda Guerra Mundial ${ }^{23}$, las formas de adquisición de territorio ${ }^{24} \mathrm{y}$ hasta el puntapié para discutir contextos que rodearon las negociaciones durante la denominada Guerra Fría ${ }^{25}$, para mencionar algunos.

De este modo, la diversidad y riqueza de los acontecimientos narrados en cada uno de los episodios ${ }^{26}$, potenciados por la periódica difusión durante jornadas maratónicas tanto en canales de aire como en sistema de cable pago, que fomenta la repetición textual

\footnotetext{
18 "La bandera de Bart" (Bart-mangled Banner), temporada 15, episodio 21.

19 "Lisa y su amor por los árboles" (Lisa the Tree Hugger), temporada 12, episodio 4; Los Simpson: la película (The Simpsons Movie), entre otros.

20 "Homero en el Ejército" (G.I. (Annoyed Grunt)), temporada 18, episodio 5.

21 "Mypods y Dinamita" (Mypods and Bombsticks), temporada 20, episodio 7.

22 “La casita del horror II" (Treehouse of Horror II), temporada 3, episodio 7.

23 "Mi héroe, el abuelo" (Raging Abe Simpson and his Grumbling Grandson in "The Curse of the Flying Hellfish), temporada 7, episodio 22. Su trama gira alrededor de un acuerdo realizado entre los integrantes del grupo Flying Hellfish, cuyos únicos sobrevivientes son Abe Simpson y el Sr. Burns, y sólo el último superviviente de ellos recibiría las pinturas. Al final del episodio, cuando el abuelo Simpson anuncia su derecho luego de deshonrar a Burns por intentar matarle, aparece un funcionario del Departamento de Estado de EE.UU. para restituir las obras a sus legítimos dueños y evitar un problema con el gobierno de Alemania. Cabe destacar que esta situación de las obras de arte ha sido objeto de reclamos judiciales reales en tribunales de EE.UU. e incluso ha llegado al cine como la película La dama de Oro (2015), protagonizada por Helen Mirren y Ryan Reynolds.
}

${ }^{24}$ Asimismo, las consecuencias de la Segunda Guerra Mundial y algunos aspectos vinculados a la adquisición de territorios según el derecho internacional se observan en "Misión deducible", (The Trouble With Trillions), temporada 9, episodio 20. Se trata de un capítulo interesante por su riqueza de contenidos que relata la colaboración de Homero con el IRS (la agencia de recaudación similar a la AFIP en la Argentina) a través de distintas misiones. En la última de ellas se le asigna la recuperación de un billete de "un trillón de dólares" emitido por Harry Truman, destinado a la reparación de Europa luego de la Segunda Guerra Mundial y que jamás llegó a destino. Las sospechas recaen, entonces, en quien era el encargado de transportarlo: el Sr. Burns. Detenido el Sr Burns, Homero le ayuda a escapar y contactan a Smithers, quien sugiere escapar del país y una vez que advierte encontrarse en "aguas internacionales", Homero festeja lanzando unos dados al grito "ya podemos jugar". Pocos segundos después advierten la presencia de varias islas con la intención de adquirirlas y establecer un Estado con la asignación de las funciones de "Presidente" y "Vicepresidente", alentando el debate para considerar los requisitos necesarios para la configuración de un Estado y la cesión de un territorio.

${ }^{25}$ Por ejemplo, a través de la referencia a El Show de Obrero y Parásito concebida -en la ficción de Los Simpsons- en 1959 por la URSS y durante la administración de Nikita Khrushchev. Fuente: "El Drama de Krusty" (Krusty Gets Kancelled), temporada 4, episodio 22.

${ }^{26}$ Incluso llegan a incluir una breve descripción de los célebres "Modelos de Naciones Unidas" que realizan los estudiantes de educación media y también de grado universitario. Fuente: "El autobús de la muerte" (Das Bus), temporada 9, episodio 14. 
de diálogos y la eternidad de situaciones desarrolladas en aquellos-transforman a la serie en una herramienta muy rica, que permite su utilización reiterada, sin tener que variar de instrumento según el contenido.

Lo anterior tiene fundamento directo en la opinión de Eikmeier, por cuanto afirma que Los Simpson poseen el mayor potencial de la historia televisiva para vincular literatura y conocimiento, debido a su ubicación histórica en horario estelar y ser la serie de mayor duración de todos los tiempos, al punto que los estudiantes que no miran regularmente el programa están familiarizados con sus personajes principales y aquellos que se reconocen fanáticos pueden describir vívidamente los personajes y las líneas argumentales. En cuanto a su masividad, la autora señala que, después de todo, estos personajes han influido culturalmente desde principios de la década de 1990 (Eikmeier, 2008: 77-78).

La última idea constituye el eslabón necesario para llegar al segundo motivo: el profundo impacto cultural que ha causado la serie -particularmente en las generaciones más jóvenes- al punto que sólo uno de cada cuatro estadounidenses puede recordar más de una de las cinco libertades garantizadas por la Primera Enmienda, mientras que más de la mitad de los encuestados puede nombrar, al menos, a dos miembros de la familia Simpson (Devins, 2007: 1335). Idéntico Ilamado de atención realiza Chris Turner en una de las principales obras dedicadas al análisis de este verdadero fenómeno bajo el título Planet Simpson y cuyo subtítulo puede traducirse de la siguiente manera: "Cómo una obra maestra de los dibujos animados documentó una Era y definió una generación". Turner define la serie como "una fuerza cultural que, en algunos aspectos es más poderosa que el rock \& roll" y sacudió al mundo (Turner, 2004: 10).

En el particular caso de los televidentes argentinos, especialmente aquellos nacidos desde la década de 1970, la hipótesis de Turner fue incluso comprobada hasta por los propios creadores de la serie. Se trata de uno de los países en los que mayor impacto ha causado ${ }^{27}$, país en donde el fanatismo se expresa en merchandising y distintos productos publicitarios, manifestaciones artísticas e innumerables referencias directas e indirectas que pretenden demostrar la posibilidad de vincular la serie con, absolutamente, casi cualquier tema.

Al respecto, se ha afirmado con razón que Los Simpson se están convirtiendo en una fuente cultural reconocible para la evaluación de cambiantes tendencias políticas, sociales y religiosas de distintos Estados, a tal punto que los especialistas en estos estudios se dedican a analizar la relevancia social de la serie (Felmate, 2013: 223).

Este impacto y popularidad de la serie resultan de mucha utilidad pedagógica en el

\footnotetext{
${ }^{27}$ Su creador, Matt Groening, declaró en una entrevista que "Los Simpson son más populares en la Argentina que en cualquier otra parte del mundo" Fuente: https://www.clarin.com/espectaculos/matt-groening20-anos-simpson 0 S1IVeccRatl.html (consulta el 06/01/2021). Esta popularidad también se hace eco en el documental oficial producido para el 20 ㅇ aniversario de la serie.
} 
aula, atento a la facilidad con la que es posible recurrir a la memoria cultural de los estudiantes sin tener que acudir a la proyección de los fragmentos de la serie animada vinculados a los contenidos - necesaria en otros casos-, a partir de lo cual se superan las habituales dificultades o deficiencia de los recursos tecnológicos disponibles para las clases presenciales en las universidades.

Finalmente, el tercer motivo es el que justifica el carácter multifuncional de la serie y su aplicación como herramienta, ya que "funciona en muchos niveles" ${ }^{28}$ a partir de la exteriorización de una frase o referencia al episodio concreto, sin desconocer que en ocasiones es necesario formular una breve explicación o contextualización adicional del ejemplo a utilizar. En nuestra experiencia podemos afirmar que, en general -y en pocos segundos, sin importar la institución educativa-, es posible obtener prácticamente el recuerdo unánime y la total atención del auditorio.

Para clarificar esta cuestión, la referencia a un episodio o circunstancia desarrollada de la serie puede funcionar como simple disparador de debates, tales como: 1) la mención a las Naciones Unidas y al cambio de representación de la Federación de Rusia/URSS en la emisión titulada "Mi querido Capitán Simpson" ${ }^{29}$; o como elemento que ayude a comprender un determinado concepto al analizarse la superada teoría de la extraterritorialidad y la posterior codificación de los privilegios e inmunidades diplomáticos en la Convención de Viena sobre Relaciones Diplomáticas de $1961^{30}$, según se puede apreciar en el capítulo “Bart

\footnotetext{
${ }^{28}$ Efectivamente, recurrimos a la expresión formulada por Homero Simpson al repetir -una y otra vez- el cortometraje titulado Los hombres son golpeados por el fútbol, un documental que obtuvo el segundo puesto del "Festival de Cine de Springfield", dirigido, producido y protagonizado por Hans Moleman, más conocido como Juan Topo en la versión latinoamericana. Fuente: "Una estrella estrellada" (A Star Is Burns), temporada 6, episodio 18.

29 “Mi querido capitán Simpson” (Simpson Tide), temporada 9, episodio 19. En esta emisión, Homero Simpson es despedido luego de insertar una dona en el núcleo de reactor de fusión y luego de ver un anuncio en la TV decide alistarse en la Marina de los EE.UU. Luego de su graduación, Homero es enviado a las habituales simulaciones estratégicas de carácter anual que realizan las escuelas de guerra y es dispuesto como miembro en un submarino nuclear (que, según Homero, debe decirse "atómico"). Homero se gana la simpatía del Capitán Tennille, quien le deja al mando del buque para inspeccionar un tubo lanza-torpedos para, posteriormente y de manera accidental, ser lanzado al exterior por orden del propio Homero quien, en consecuencia, queda al mando y en el intento de regresar a Springfield se dirige a aguas bajo jurisdicción de la Federación Rusa, provocando un incidente en la Organización de las Naciones Unidas. En comentario adicional, el capítulo constituye una parodia de la película norteamericana Marea roja (Crimson Tide, 1995), protagonizada por Denzel Washington y Gene Hackman.

${ }^{30}$ El artículo 1.i) de la Convención define como "local de la misión" a los edificios o las partes de los edificios, sea cual fuere su propietario, utilizados para las finalidades de la misión, incluyendo la residencia del jefe de la misión , así como el terreno destinado al servicio de esos edificios o de parte de ellos". Cfr. Convención de Viena sobre Relaciones Diplomáticas de 1961, adoptada el 18 de abril de 1961, United Nations Treaty Series, Vol. 500, artículo 1 (i). Al respecto Arredondo sostiene que los locales de la misión comprenden todas aquellas oficinas que forman parte de lo que se denomina la Cancillería y también la "residencia" del Jefe de misión diplomática (Arredondo, 2016: 97).
} 
contra Australia" ${ }^{31}$, o bien como objeto de un análisis más complejo, que es lo que desarrollaremos a continuación.

\section{El recurso a Los Simpson para explicar el régimen jurídico de la alta mar: un test exitoso}

Entre las numerosas transmisiones de Los Simpson que resultan aptas para la enseñanza del derecho internacional, hemos seleccionado un episodio que -por su extensiónpermite formular un amplio desarrollo de los contenidos e incluso -si se lo propone como actividad- organizar toda una clase a su alrededor. Asimismo, como se verá, se trata de un ejemplo que ha sido utilizado por ambos autores con resultado exitoso en universidades ubicadas en la provincia de Córdoba y en la Ciudad de Buenos Aires.

Se trata del episodio titulado "La familia Mansión", perteneciente a la temporada 11 de la serie. La historia se inicia a raíz de un estudio médico que debe realizarse el Sr. Burns en la Clínica Mayo, en Minnesota, y que le exige dejar su mansión al cuidado de Homero Simpson y su familia. Naturalmente, la situación provoca que Homero comience a vivir como un millonario y que, para ostentarlo, invite a sus amigos a una fiesta organizada en el yate de Burns, el Gone Fission. Este hecho es alimentado por el cantinero Moe, quien informa a Homero que no podía venderle cerveza hasta las 14:00 horas, ya que era día domingo -y Burns regresaba al día siguiente-, a menos que fueran a "aguas internacionales, el océano situado a 19 kilómetros de la costa", una zona que el propio Homero interpreta como "alta mar".

Al alejarse más allá del mar territorial de los EE.UU. -hecho señalado por unas boyas flotantes y carteles que así lo anuncian en el episodio-, Homero afirma encontrarse en "aguas internacionales" y la caracteriza como "el lugar que la ley olvidó".

Las situaciones hilarantes en alta mar no se hacen esperar e incluyen numerosos sucesos absurdos como fiestas, corridas de toros, ajustes mafiosos, transmisiones de partidos con "consentimiento oral no expresado por escrito", el matrimonio de un individuo con un rumiante, utilización de fuegos de artificios ilegales y peleas de monos armados con elementos cortantes, ya que, tal como afirma Bart, en esta zona "se puede hacer de todo". Los sucesos decantan, como no podía ser de otra manera, en la abrupta interrupción de la fiesta por parte de un ataque de piratas provenientes de Hong-Kong.

No creemos necesario profundizar más, ya que el internacionalista avezado en la asignatura -y en Los Simpson- puede advertir claramente la utilidad del episodio para el explicar los conceptos de un punto trascendental de la asignatura: el régimen jurídico de la alta mar. En su contenido, el capítulo incluye una sutil caracterización de ésta como un lugar no sujeto

31 "Bart contra Australia" (Bart vs. Australia), temporada 6, episodio 16. 
a la jurisdicción estatal -el "lugar que la ley olvidó", según Homero, como señalamos anteriormente-, aunque también se refiere a la realización de prácticas prohibidas, como las emisiones no autorizadas ${ }^{32}$ (ilustradas por la presencia de un buque que retransmite los partidos de béisbol de las Ligas Mayores ${ }^{33}$ ); y la propia piratería, según la definición dispuesta en el artículo 101 de la Convención de las Naciones Unidas sobre el Derecho del Mar de $1982^{34}$.

La riqueza en contenido del episodio permite así dedicar al tema una clase, estructurada en tres secciones:

1) Sección inicial: la utilización de los fragmentos del episodio -debidamente editados y proyectados- funciona como "disparador de debate" y permite identificar, mediante el diálogo con los estudiantes, los aspectos más relevantes del tema a la luz de lo ocurrido en el capítulo y las líneas de los personajes ${ }^{35}$;

2) Sección intermedia: necesariamente presencial y de carácter expositivo, consiste en debatir sobre los aciertos y errores jurídicos que presenta el capítulo. Se trata de un ejercicio de construcción de conocimiento a través del método socrático, empleando el diálogo para referirse pormenorizadamente a las normas de la Convención de las Naciones Unidas sobre el Derecho del Mar de 1982 aplicable al caso en cuestión; $y$,

3) Sección final: constituida por una etapa de evaluación en el que se proponen dos casos prácticos con el objeto de aunar los aspectos identificados en la primera parte y desarrollados en la segunda ${ }^{36}$. Ello permite no solamente conocer el grado de asimilación de los contenidos, sino también evaluar la precisión jurídica del episodio objeto de análisis.

${ }^{32}$ Cfr. Convención de las Naciones Unidas sobre el Derecho del Mar, adoptada el 30 de abril de 1982, United Nations Treaty Series, Vol. 1833, artículo 109.

\footnotetext{
${ }^{33}$ Se trata de la Major League Baseball, más conocida como MLB, la organización más importante que nuclea al béisbol profesional de los EE.UU., repartidos entre la Liga Nacional y la Liga Americana, cuyos campeones se enfrentan posteriormente en la denominada "Serie Mundial".
}

${ }^{34}$ Cfr. Convención de las Naciones Unidas sobre el Derecho del Mar, adoptada el 30 de abril de 1982, United Nations Treaty Series, Vol. 1833, artículo 109. Sin embargo, Gutoff advierte que Homero dirige al Gone Fission -el yate de Burns- más allá de las 12 millas y, por esta razón no se trataría de la alta mar, sino de la ZEE, al expresar que "Homer decides to throw a party aboard Gone Fission, Mr. Bums' yacht, and pilots her to outside the 12-mile limit so as to be able to buy beer in the morning, contrary to state law..." (Gutoff, 2000: 647). No obstante tener razón, creemos que el fundamento de la solución se encuentra en la naturaleza de la ZEE, un espacio sui generis que no es alta mar ni mar territorial, pero que habilita la represión de la piratería en esta zona bajo jurisdicción universal, razón por la cual el argumento de la Guardia Costera de EE.UU. durante el episodio -para realizar una acción más allá de las 12 millas- no resulta jurídicamente válido, ya que podría haber auxiliado al yate Gone Fission (Godio, 2012: 542). Para profundizar sobre este tema ver ConSIGLI, J. A. (coord.) (2011). Piratería en el siglo XXI: nuevos escenarios para el derecho internacional, Buenos Aires, Consejo Argentino para las Relaciones Internacionales.

${ }^{35}$ A fin de aprovechar el tiempo de trabajo en el aula, se puede indicar a los alumnos que consulten el capítulo
con antelación y dedicar un plazo mayor a las etapas y actividades siguientes, de carácter presencial.

${ }^{36}$ Concretamente, los casos tienen por objeto identificar la retransmisión de los partidos de béisbol con las 
Sobre esta experiencia es necesario señalar que no sólo posee un singular éxito como actividad, sino que ha generado el aporte espontáneo de los estudiantes al referenciar otras escenas, reflexiones y recuerdos sobre los episodios que, en definitiva, corroboran nuestra afirmación inicial conforme la cual la técnica propuesta permite también a los alumnos asociar contenidos propios del derecho internacional a su propio acervo cultural "simpsoniano".

\section{Consideraciones finales}

Durante el desarrollo del presente trabajo hemos identificado y demostrado la conveniencia de utilizar referencias de la cultura popular masiva como herramienta didáctica idónea para la enseñanza del derecho internacional. En ese sentido la serie animada Los Simpson constituye un uso justificado en la implementación de un diseño alternativo metodológico que funciona como un "disparador" efectivo para la síntesis de lecturas obligatorias, el debate y otras estrategias colaborativas, cuya implementación permite reflexionar más allá de los límites espacio-temporales del aula convencional y -además, cuestión no es menor- permitir la confección de un diseño de clase realmente orientado a partir de la participación del alumno con un elevado nivel de retroalimentación (feedback), prácticamente garantizado.

Por otra parte, a partir de los fundamentos teóricos esbozados y la experiencia práctica de testeo exitosa -tanto en condiciones experimentales, como de aplicación de campo, hemos demostrado que técnica propuesta constituye una alternativa metodológica viable para la enseñanza del derecho internacional. Esto, por supuesto, no significa que nuestras ideas garanticen un escenario áulico con un $100 \%$ de éxito para todos los estudiantes, debido a la presencia de algunos alumnos que no son fanáticos de Los Simpsons. Sin embargo, la práctica omnipresencia de la serie animada en la vida cotidiana permite simplemente, como se sostuvo durante el desarrollo del trabajo, evocar la memoria de los estudiantes para obtener un resultado en promedio más que positivo.

Incluso pensamos que el desarrollo ulterior de esta técnica, a partir de su difusión entre colegas y profundizando su uso, puede redundar en la elaboración de un programa completo de clases vinculadas a temas tratados por Los Simpson e incentivar actividades extracurriculares, como talleres o seminarios destinados no solamente a utilizar el recurso instrumentalmente como vía de transmisión de contenidos, sino también a estimular un análisis crítico de las tramas y los personajes desde la mirada del derecho internacional.

Se ha dicho que Los Simpson contienen al menos un ejemplo para cada situación de la vida, tal como ocurre con las inquietantes preguntas que recibimos los profesores en cada curso, como "lo que nos enseñan, ¿sirve para algo en la vida real?” o la clásica "¿podría repetir la pregunta? No estaba escuchando" y que Matt Groening supo señalar en uno de

\footnotetext{
"transmisiones no autorizadas" y el análisis de la estrategia sugerida por Moe -desarrollada por Homero- de navegar con el yate hasta la alta mar para eludir la aplicación de los reglamentos sobre bebidas alcohólicas. Cfr. Convención de las Naciones Unidas sobre el Derecho del Mar, adoptada el 30 de abril de 1982, United Nations Treaty Series, Vol. 1833, artículos 94 y 109.
} 
sus textos (Groening, 1993: 15). Del mismo modo, puede ocurrir que hayamos omitido involuntariamente algunos episodios, aunque confiamos en que nuestros lectores y alumnos, sin duda fanáticos de esta serie -que invita a una "repetición de visualización" para repasar los episodios con el fin de recoger referencias omitidas anteriormente- nos señalen aquellas que resulten pertinentes a este trabajo. Sin embargo, creemos que hemos realizado una labor suficiente para demostrar que el derecho internacional público posee numerosas referencias en la serie, las que, entendemos, justifican ampliamente la utilización de la estrategia propuesta.

Finalmente, resta señalar que la referencia al título de nuestro trabajo no constituye más que un homenaje al ficticio abogado Lionel Hutz y su conocida frase: “(...) lo que más necesita este país es más abogados. ¿Te imaginas al mundo sin abogados?". Habitualmente, la interpretación cultural de las palabras de Hutz giran en rededor de la avidez y el pleito litigioso que peyorativamente comprende al personaje, pero lo cierto es que también podría considerarse como un mensaje hacia nuestros contemporáneos y afirmar que toda sociedad saludable -o armoniosa, si evocamos la misma imagen del abogado Hutz-requiere que sus ciudadanos conozcan, entiendan y respeten la Ley, por lo que todos debemos tener una cuota del arte de abogar por lo justo y velar por ello o bien, de no ser esto posible, conformarse con ocho dólares la hora, dos paletas del refrigerador y una jaula vieja, porque, al fin y al cabo, somos una amenaza.

\section{Bibliografía}

- ArRedondo, R. (2016). Derecho Diplomático y Consular, Abeledo-Perrot.

- Berasategul, V.E. (2011). Malvinas. Diplomacia y Conflicto Armado, Proa.

- Bergoglio, M. I. (2006). "Las facultades de derecho argentinas entre las tradiciones y los esfuerzos de cambio", en R. Pérez Perdomo \& J. Rodríguez Torres (comp.), La formación jurídica en América Latina, Universidad Externado de Colombia, pp. 101-130.

- BöHMER, M. F. (2003). "Algunas sugerencias para escapar del silencio del aula”, Academia. Revista sobre enseñanza del Derecho de Buenos Aires, año 1, n 1, pp. 13-34.

- ConsIGLI, J. A. (coord.) (2011). Piratería en el siglo XXI: nuevos escenarios para el derecho internacional, Consejo Argentino para las Relaciones Internacionales.

- CozzettI, M. (2011). “Bart, el coleccionista”, El Reverso, no 8, pp. 4-5.

- Devins, N. (2007). “The D'Oh! of Popular Constitutionalism”, Michigan Law Review, v. 105, n 6, pp. 1333-1351.

- EIKMEIER, G. M. (2008). “D'oh! Using 'The Simpsons' to Improve Student Response to Literature", The English Journal, v. 97, n² 4, pp. 77-80.

- Felmate, D. (2013). "It's Funny Because It's True? The Simpsons, Satire, and the Significance of Religious Humor in Popular Culture", Journal of the American Academy of Religion, v. 81, $n^{\circ} 1$, pp. 222-248. 
- Fucito, F. (2000), El profesor de derecho en las universidades de Buenos Aires y Nacional de La Plata. Un estudio comparativo, Editorial de la Universidad Nacional de La Plata.

- Godio, L. M. A. (2012). “El Derecho del Mar”, en R. Arredondo (dir.), Manual de Derecho Internacional Público, La Ley, pp. 523-558.

- Groening, M. (ed.) (1993). Bart Simpson's Guide to Life: A Wee Handbook for the Perplexed, HarperCollins Publisher.

- Gutoff, J. M. (2000). "The Law of Piracy in Popular Culture", Journal of Maritime Law and Commerce, v. 31, $n^{\circ} 4$, pp. 643-648.

- Ho, K. K. (2003). "The Simpsons and the Law: Revealing Truth and Justice to the Masses", UCLA Entertainment Law Review, v. 10, n², pp. 275-288.

- IrWin, W., Conard, M. T. \& Skoblle, A. J. (2009). Los Simpson y la Filosofía, Blackie Books.

- JAMneJAD, M. \& Wood, M. (2009). "The Principle of Non-intervention", Leiden Journal of International Law, v. 22, pp. 345-381.

- KeSlowitz, S. (2008). "The Simpsons, 24, and the Law: How Homer Simpson and Jack Bauer Influence Congressional Lawmaking and Judicial Reasoning", Cardozo Law Review, v. 29, pp. 2787-2822.

- Kohen, M. G. \& Rodríguez, F. D. (2015). Las Malvinas entre el Derecho y la Historia, Eudeba.

- LISTA, C. A. (2006), "La educación jurídica en Argentina: tendencias y perspectivas”, en R. Pérez Perdomo \& J. Rodríguez Torres (comp.), La formación jurídica en América Latina, Universidad Externado de Colombia, pp. 237-273.

- LUCCASEN, R. A. \& ThOMAS, M. K. (2010). “Simpsonomics: Teaching Economics Using Episodes of 'The Simpsons'", The Journal of Economic Education, v. 41, n’ 2, pp. 136149.

- SÁnCHEZ, C. H. (2010), Todo lo que sé de ciencia lo aprendí mirando Los Simpson, Ed. del autor.

- SCANLAN, S. J. \& Feinberg, S. L. (2000), “The Cartoon Society: Using 'The Simpsons' to Teach and Learn Sociology", Teaching Sociology, v. 28, n² 2, pp. 127-139.

- TerReLL, S. (2014/2015). “LOL Teacher! Using humor to enhance student learning”, Educational Horizons, v. 93, $\mathrm{n}^{\circ}$ 2, pp. 6-7.

- TURneR, C. (2004). Planet Simpson: How a Cartoon Masterpiece Documented an Era and Defined a Generation, Random House. 